\title{
On the Bifurcation Set of a Polynomial Function and Newton Boundary
}

\author{
By
}

\author{
András NémeThi* and Alexandru ZAHARIA*
}

\section{§1. Introduction}

Let $f: \mathbb{C}^{n} \rightarrow \boldsymbol{C}$ be a polynomial function. It is well known that there exists a finite set $\Gamma \subseteq C$, such that $f: C^{n} \backslash f^{-1}(\Gamma) \rightarrow C \backslash \Gamma$ is a locally trivial fibration (see [1], [5], [13], [15], [16]). The smallest such set $\Gamma$ we call the bifurcation set, denoted by $B_{f}$ (in [1], [2] it is called the set of atypical values). Since the map $f$ is not proper, the set $B_{f}$ contains besides the set $\Sigma_{f}$ of all critical values of $f$ perhaps some other points (the "critical values at infinity" or "critical values of second type" [12]). There are some special cases when the polynomial has no critical values at infinity (hence $B_{f}=\Sigma_{f}$ ): Pham [13] and Fedoryuk [4] have imposed lowerbound conditions for $\|\operatorname{grad} f(x)\|$ for large values of $\|x\|$, Kouchnirenko has proved in [6] for convenient polynomials with nondegenerate Newton principal part at infinity, Broughton [1], [2] for "tame" polynomials and the first author [8], [9] for the larger class of "quasitame" polynomials.

In this note we give an explicit set $S_{f}$, such that $B_{f} \leqq \Sigma_{f} \cup S_{f}$. More precisely, let $\left.\operatorname{grad} f(z)=\overline{\left(\frac{\partial f}{\partial z_{1}}(z)\right.}, \cdots, \overline{\frac{\partial f}{\partial z_{n}}(z)}\right)$. We denote by $\mathscr{M}(f)$ the Milnor set of the polynomial $f$, namely

$$
\mathscr{M}(f)=\left\{z \in \boldsymbol{C}^{n} ; \text { there exists } \lambda \in \boldsymbol{C} \text { such that } \operatorname{grad} f(z)=\lambda z\right\} .
$$

We define the set $S_{f}$ by :

$$
S_{f}=\left\{\begin{array}{l}
c \in C ; \text { there exists a sequence }\left\{z^{k}\right\}_{k} \subseteq M(f) \text { such that } \\
\lim _{k \rightarrow \infty}\left\|z^{k}\right\|=\infty \text { and } \lim _{k \rightarrow \infty} f\left(z^{k}\right)=c
\end{array}\right\}
$$

In the second section we prove:

Theorem 1. Let $f: C^{n} \rightarrow C$ be a polynomial map. Then $B_{f} \leqq \Sigma_{f} \cup S_{f}$.

Communicated by K. Saito, December 25, 1989.

* Department of Mathematics INCREST, Bd. Păcii 220, RO-79622 Bucharest, Romania. 
In the third section we consider the Newton nondegenerate case. In this situation, the set $S_{f}$ can be determined very easy and explicitly. Namely, let $f=\sum_{\nu \in N} a_{\nu} z^{\nu}$ be a polynomial of $n$ variables (where $\nu=\left(\nu_{1}, \cdots, \nu_{n}\right)$ and $z^{\nu}=z_{1}^{\nu} \ldots$. $z_{n}^{\nu n}$ as usual) with $f(0)=0$. As in [6], [11] we denote $\operatorname{supp}(f)=\left\{\nu \in N^{n} ; a_{\nu} \neq 0\right\}$, $\overline{\operatorname{supp}(f)}=$ the convex closure in $\boldsymbol{R}^{n}$ of $\operatorname{supp}(f), \tilde{\Gamma}_{-}(f)=$ the convex closure of $\{0\} \cup \operatorname{supp}(f), \tilde{\Gamma}(f)=$ the union of the closed faces of the polyhedron $\tilde{\Gamma}_{-}(f)$ which do not contain the origin. If $\Delta \subseteq \tilde{\Gamma}(f)$ is a closed face, we note $f_{\Delta}(z)=\sum_{\nu \in \Delta} a_{\nu} z^{\nu}$ and we say that $f$ is nondegenerate on $\Delta$ if the system of equations $\frac{\partial f_{\Delta}}{\partial z_{1}}(z)=\cdots=\frac{\partial f_{\Delta}}{\partial z_{n}}(z)=0$ has no solutions in $\left(\boldsymbol{C}^{*}\right)^{n}$. We say that $f$ is Newton nondegenerate if for every compact face $\Delta$ of $\tilde{\Gamma}(f), f$ is nondegenerate on $\Delta$. By definition, $f$ is convenient if the intersection of $\operatorname{supp}(f)$ with each coordinate axis is non-empty.

A closed face $\Delta \subseteq \overline{\operatorname{supp}(f)}$ is called bad if :

(i) the affine subvariety of $\operatorname{dimension}=\operatorname{dim} \Delta$ spaned by $\Delta$ contains the origin, and

(ii) there exists a hyperplane $H \subseteq \boldsymbol{R}^{n}$ with equation $a_{1} x_{1}+\cdots+a_{n} x_{n}=0$ (where $x_{1}, \cdots, x_{n}$ are the coordinates in $\boldsymbol{R}^{n}$ ) such that
a) there exist $i$ and $j$ with $a_{i}<0$ and $a_{j}>0$
b) $H \cap \overline{\operatorname{supp}(f)}=\Delta$.

We can express more geometrically the condition (iia) by saying that the hyperplane $H$ intersects the interior of the positive octant $\left(\boldsymbol{R}_{+}\right)^{n}$.

Let $\mathscr{B}$ denote the set of bad faces of $\overline{\operatorname{supp}(f)}$. If $\Delta \in \mathscr{B}$ we define :

$$
\Sigma_{\Delta}=\left\{f_{\Delta}\left(z^{0}\right) ; z^{0} \in\left(C^{*}\right)^{n} \text { and } \operatorname{grad} f_{\Delta}\left(z^{0}\right)=0\right\} .
$$

It is clear that $\Sigma_{\Delta} \leqq \Sigma_{f_{\Delta}}$, hence $\Sigma_{\Delta}$ is a finite set.

We have the following:

Theorem 2. Suppose that $f$ is not convenient, Newton nondegenerate and $f(0)=0$. Then $B_{f} \subseteq \Sigma_{f} \cup\{0\} \cup \bigcup_{\Delta \in \mathscr{B}} \Sigma_{\Delta}$.

In the convenient case, $B_{f}=\Sigma_{f}$ (see [6], [2]).

In the last section of this note there are some remarks. Also, for $n=2$, we compare our Theorem 2 with the result of Hà and Lê, which gives $B_{f}$ in terms of the Euler-characteristic of the fibers $f^{-1}(c)$ (see [5]). In particular, for $n=2$, our formula is the best possible (see Proposition 6).

\section{$\S 2$. Proof of Theorem 1}

We need the following lemma, which is a direct consequence of the definitions : 
Lemma 3. Let $D \subseteq C \backslash S_{f}$ be a closed disc. Then $f^{-1}(D) \cap \mathscr{M}(f)$ is bounded.

For $a, b \in C^{n}$ we note $\langle a, b\rangle=\sum_{j=1}^{n} a, \bar{b}_{\jmath}$. For $R \in(0, \infty)$ we put $S_{R}=\left\{z \in \boldsymbol{C}^{n}\right.$; $\|z\|=R\}$ and $B_{R}=\left\{z \in C^{n} ;\|z\| \leqq R\right\}$.

Now for the proof of the theorem we fix $c \in C \backslash\left(\Sigma_{f} \cup S_{f}\right)$ and $D$ a small open disc centered at $c$, with the closure $\bar{D} \subseteq C \backslash\left(\Sigma_{f} \cup S_{f}\right)$. Let $R \in(0, \infty)$ be sufficiently large such that $f^{-1}(D) \cap \mathscr{M}(f) \cap\left\{z \in C^{n} ;\|z\| \geqq R\right\}=\phi$ (this is possible by Lemma 3 ) and such that $S_{R}$ meets transversally $f^{-1}\left(c^{\prime}\right)$ for all $c^{\prime} \in D$ (also possible if $D$ is small enough). It follows that $\operatorname{grad} f(z)$ and $z$ are $C$-linearly independent vectors for all $z \in A$, where $A=f^{-1}(D) \cap\left\{z \in C^{n} ;\|z\| \geqq R\right\}$, and therefore we can find a smooth vector field $v_{1}(z)$ on $A$ such that $\left\langle v_{1}(z), z\right\rangle=0$ and $\left\langle v_{1}(z), \operatorname{grad} f(z)\right\rangle=1$.

Let $\varepsilon>0$ be such that for every $R^{\prime} \in[R, R+\varepsilon]$ and for every $d \in D$, we have $f^{-1}(d) \cap S_{R^{\prime}}$. Since $D \cap \Sigma_{f}=\phi$, the fibration theorem of Ehresmann gives that the restriction $f:\left(f^{-1}(D) \cap B_{R+\varepsilon}, f^{-1}(D) \cap S_{R+\varepsilon}\right) \rightarrow D$ is a locally trivial fibration with the fiber $F=f^{-1}(c) \cap B_{R+\varepsilon}$, a smooth manifold with boundary $\partial F=f^{-1}(c) \cap S_{R+\varepsilon}$. Hence there exists a diffeomorphism $\phi:(F \times D, \partial F \times D) \rightarrow\left(f^{-1}(D) \cap B_{R+\varepsilon}, f^{-1}(D) \cap\right.$ $S_{R+\varepsilon}$ ) such that $f \circ \phi$ is the projection onto $D$. Thus the vector field $w: F \times D$ $\rightarrow T(F \times D)=T F \times T D, w(z, d)=((z, 0),(d, 1))$ will give a vector field $v_{2}$ on $f^{-1}(D) \cap B_{R+\varepsilon}$ such that $\left\langle v_{2}(z), \operatorname{grad} f(z)\right\rangle=1$ for every $z$. Glueing together $v_{1}$ and $v_{2}$, we obtain a vector field $v$ on $f^{-1}(D)$ such that $\langle v, \operatorname{grad} f\rangle=1$ and such that for every $z$ with $\|z\| \geqq R+\varepsilon$, we have $\langle v(z), z\rangle=0$. Now using the solutions of the differential equation $\frac{d z}{d t}=v(z)$ we obtain that the restriction $f: f^{-1}(D)$ $\rightarrow D$ is a trivial fibration.

\section{§3. Proof of Theorem 2}

We need a version of Curve Selection Lemma from Milnor's book [7]. This seems to be well known. For a proof, we refer the reader to [10].

Lemma 4. (Curve Selection Lemma) Let $f_{1}, \cdots, f_{q}, g_{1}, \cdots, g_{s}, h_{1}, \cdots, h_{r}$ $\in \boldsymbol{R}\left[X_{1}, \cdots, X_{m}\right]$ be polynomial functions with real coefficients. Let $U=\left\{x \in \boldsymbol{R}^{m}\right.$; $\left.f_{2}(x)=0, i=1, \cdots, q\right\}$ and $W=\left\{x \in \boldsymbol{R}^{m} ; g_{i}(x)>0, i=1, \cdots, s\right\}$. Suppose that there exists a sequence $\left\{x^{k}\right\} \subseteq U \cap W$ such that $\lim _{k \rightarrow \infty}\left\|x^{k}\right\|=\infty$ and for all $j \in\{1, \cdots, r\}$, $\lim _{k \rightarrow \infty} h_{j}\left(x^{k}\right)=0$. Then there exists a real analytic curve $p:(0, \varepsilon) \rightarrow U \cap W$ with $\lim _{t \rightarrow 0}\|p(t)\|=\infty, \lim _{t \rightarrow 0} h_{\jmath}(p(t))=0$ for $1 \leqq j \leqq r$ and of the form $p(t)=a t^{a}+a_{1} t^{a+1}+\cdots$ with $a \in \boldsymbol{R}^{m} \backslash\{0\}$ and $\alpha<0$.

Using Theorem 1 and Curve Selection Lemma, it is sufficient to prove the following : 
If $f$ is a Newton nondegenerate polynomial with $f(0)=0$ and if $p(t) \in \mathscr{M}(f)$ is an analytic curve such that

$$
\lim _{t \rightarrow 0}\|p(t)\|=\infty \quad \text { and } \quad \lim _{t \rightarrow 0} f(p(t)) \in C
$$

then $\lim _{t \rightarrow 0} f(p(t)) \in \Sigma_{f} \cup\{0\} \cup \bigcup_{\Delta \in \mathscr{B}} \Sigma_{\Delta}$.

To prove this we consider the expansions

$$
\left\{\begin{array}{l}
p(t)=a t^{\alpha}+a_{1} t^{\alpha+1}+\cdots \\
f(p(t))=b t^{\beta}+b_{1} t^{\beta+1}+\cdots \\
\operatorname{grad} f(p(t))=c t^{\gamma}+c_{1} t^{\gamma+1}+\cdots
\end{array}\right.
$$

and the identity

$$
\frac{d f(p(t))}{d t}=\left\langle\frac{d p}{d t}, \operatorname{grad} f(p(t))\right\rangle
$$

The condition $p(t) \in \mathscr{M}(f)$ means that there exists an analytic curve $\lambda(t) \in \boldsymbol{C}$ such that for every $t$, we have

$$
\operatorname{grad} f(p(t))=\lambda(t) p(t) .
$$

If $\operatorname{grad} f(p(t)) \equiv 0$, the identity (3) shows that $f(p(t))$ is constant with respect to $t$, namely $f(p(t)) \in \Sigma_{f}$.

So we can suppose that $\operatorname{grad} f(p(t)) \not \equiv 0$. Similarly, $f(p(t)) \not \equiv 0$, since otherwise by derivation $\lambda(t) \cdot\left\langle\frac{d p}{d t}, p\right\rangle=0$ which is in contradiction with $\operatorname{grad} f(p(t))$ $\not \equiv 0$ and $\left\langle\frac{d p}{d t}, p\right\rangle \equiv 0$. From (4) we get also $\lambda(t) \neq 0$. Let $\lambda(t)=\lambda_{0} t^{\delta}+\lambda_{1} t^{\delta+1}+\cdots$ be the expansion of $\lambda(t)$, where $\lambda_{0} \neq 0$. From (1) we can assume that $a \neq 0, \alpha<0$, $b \neq 0, \beta \geqq 0$ and $c \neq 0$. Using (4), the scalar product $\langle a, c\rangle \neq 0$, hence from the expansions (2) and the formula (3) we get that $\gamma+\alpha-1 \geqq 0$ and thus $\gamma>0$.

Renumbering the] coordinates, if necessary, we may assume that $p(t)=$ $\left(p_{1}(t), \cdots, p_{n}(t)\right)=\left(w_{1}^{0} t^{\nu_{1}}+w_{1}^{1} t^{\nu_{1}+1}+\cdots, \cdots, w_{k}^{0} t^{\nu_{k}}+w_{k}^{1} t^{\nu_{k}+1}+\cdots, 0, \cdots, 0\right)$, where $w_{1}^{0} \neq 0, \cdots, w_{k}^{0} \neq 0$ and $\alpha=\nu_{1} \leqq \nu_{2} \leqq \cdots \leqq \nu_{k}$. Identifying $\boldsymbol{R}^{k}$ with $\left\{x \in \boldsymbol{R}^{n}\right.$; $\left.x_{k+1}=\cdots=x_{n}=0\right\}$ we have $\operatorname{supp}(f) \cap \boldsymbol{R}^{k} \neq \phi$ since $f(p(t)) \neq \equiv$.

Consider the continuous function $l_{\nu}(x)=\sum_{j=1}^{k} \nu_{j} x_{j}$ on $\boldsymbol{R}^{n}$. Let $\Delta$ be the unique face of $\overline{\operatorname{supp}(f)} \cap \boldsymbol{R}^{k}$ where the restriction $l_{\nu}: \overline{\operatorname{supp}(f)} \cap \boldsymbol{R}^{k} \rightarrow \boldsymbol{R}$ takes the minimal value, say $d$, and let $m \in(-\infty, 0)$ be such that

$$
m<\min \left\{l_{\nu}(x) ; x=\overline{\operatorname{supp}(f)}\right\} \text {. }
$$
Then $f(p(t))=f_{\Delta}\left(w_{0}^{0}\right) t^{d}+\cdots$, and for $j=1, \cdots, k, \frac{\partial f}{\partial z_{j}}(p(t))=\frac{\partial f_{\Delta}}{\partial z_{j}}\left(w^{0}\right) t^{d-\nu_{j}}+\cdots$,
where $w^{0}=\left(w_{1}^{0}, \cdots, w_{k}^{0}, 1, \cdots, 1\right)$. 
If $d>0$, we have $\lim _{t \rightarrow 0} f(p(t))=0$.

If $d=0$ and $\nu_{k} \leqq 0$, then $f\left(z_{1}, \cdots, z_{k}, 0, \cdots, 0\right)$ does not depend on $z_{1}$, hence $\frac{\partial f}{\partial z_{1}}(p(t)) \equiv 0$, in contradiction with $(4)$ and $p_{1}(t) \neq \equiv$.

If $d=0$ and $\nu_{k}>0$, then for the hyperplane $H \subseteq R^{n}$ with equation $\nu_{1} x_{1}+\cdots$ $+\nu_{k} x_{k}-m\left(x_{k+1}+\cdots+x_{n}\right)=0$ and the face $\Delta$, the condition (ii) is fulfilled. Thus if (i) is not fulfilled, then $\Delta$ is not a bad face of $\overline{\operatorname{supp}(f)}$ and it follows that $\Delta$ is a closed face of $\tilde{\Gamma}(f)$. By the nondegeneracy condition on $\Delta$, there exists $l \in\{1, \cdots, k\}$ such that $\frac{\partial f_{\Delta}}{\partial z_{l}}\left(w^{0}\right) \neq 0$. (We recall that $f_{\Delta}(z)$ does not depend on the variables $z_{k+1}, \cdots, z_{n}$.)

But this is in contradiction with the following lemma:

Lemma 5. Lei $d, \Delta, w^{0}$ be defined as above. Suppose that $d \leqq 0$ and $d \cdot f_{\Delta}\left(w^{0}\right)=0$. Then there exists no $l \subseteq\{1, \cdots, k\}$ such that $\frac{\partial f_{\Delta}}{\partial z_{l}}\left(w^{0}\right) \neq 0$.

Proof of Lemma 5. Suppose that there exists $l \in\{1, \cdots, k\}$ such that $\frac{\partial f_{\Delta}}{\partial z_{l}}\left(w^{0}\right) \neq 0$. By condition (4) and $\gamma>0$ we get that $\delta+\nu_{l}=d-\nu_{l}>0$, hence $\nu_{l}<0$. Let $I=\left\{j ; \nu_{j}=\nu_{l}\right\}$. Again by (4) we have for $j \in\{1, \cdots, k\}$ :

$$
\begin{aligned}
& j \in I \Longrightarrow d-\nu_{\jmath}=\delta+\nu_{\jmath} \text { and } \frac{\partial f_{\Delta}}{\partial z_{j}}\left(w^{0}\right)=\bar{\lambda}_{0} \overline{w_{\jmath}^{0}} ; \\
& j \notin I \Longrightarrow d-\nu_{\jmath}<\delta+\nu_{\jmath}, \text { hence } \frac{\partial f_{\Delta}}{\partial z_{j}}\left(w^{0}\right)=0 .
\end{aligned}
$$

Thus, from the Euler relation for the weakly quasihomogeneous polynomial $f_{\Delta}$, $\sum_{j=1}^{k} \nu_{\jmath} z_{\jmath} \frac{\partial f_{\Delta}}{\partial z_{j}}(z)=d \cdot f_{\Delta}(z)$, we obtain for $z=w^{0}$ the absurd equality $\nu_{l} \cdot \lambda_{0} \sum_{j \in I}\left|w_{\jmath}^{0}\right|^{2}=0$. This ends the proof of Lemma 5 .

It follows that (i) is also fulfilled and $\Delta \in \mathscr{B}$. By the above lemma, $\frac{\partial f_{\Delta}}{\partial z_{l}}\left(w^{0}\right)=0$ for all $l \in\{1, \cdots, k\}$ and thus we have $\lim _{t \rightarrow 0} f(p(t))=f_{\Delta}\left(w^{0}\right) \in \Sigma_{\Delta}$.

It remains to consider the case $d<0$. With this assumption it follows that $\Delta$ is a closed face of $\tilde{\Gamma}(f)$. Since $\beta \geqq 0$ we get $f_{d}\left(w^{0}\right)=0$, hence the nondegeneracy condition on $\Delta$ is in contradiction with the above lemma.

\section{§4. Some Remarks}

1. S. A. Broughton described in [1] and [2] the class $\subseteq$ of tame polynomials and proved that for a tame polynomial $f$, we have $B_{f}=\Sigma_{f}$.

In [8] and [9], the first author considered the class $Q I$ of quasitame poly- 
nomials and also proved that $B_{f}=\Sigma_{f}$ for any quasitame polynomial $f$. If we denote by $\mathscr{M} I$ the class of $\mathscr{M}$-tame polynomials, namely the polynomials $f$ with $S_{f}=\phi$, then $\mathscr{I} \subseteq Q \mathscr{I} \subseteq \mathscr{M I}$, the first inclusion being strict. We don't know if the second inclusion is an equality or not. For other interesting properties of these classes of polynomials see also [10].

2. Also in [8] and [9], the first author proved that $B_{f} \cong \Lambda_{f}$, where

$$
\Lambda_{f}:=\left\{\begin{array}{l}
c \in C ; \text { there exists a sequence }\left\{z^{k}\right\}_{k} \in C^{n} \text { such that } \lim _{k \rightarrow \infty} \operatorname{grad} f\left(z^{k}\right)=0 \\
\text { and } \lim _{k \rightarrow \infty}\left(f\left(z^{k}\right)-\left\langle z^{k}, \operatorname{grad} f\left(z^{k}\right)\right\rangle\right)=c
\end{array}\right\}
$$

It is not hard to prove that $\Sigma_{f} \cup S_{f} \subseteq \Lambda_{f}$, but in general we have no equality, hence the set $\Sigma_{f} \cup S_{f}$ is a better approximation for $B_{f}$. Such an example is the polynomial $f=x^{5} y^{3}+x^{5} z^{2}+x^{11} y^{3} z^{2}+x$ which is Newton nondegenerate (hence $\Sigma_{f} \cup S_{f}$ is a finite set) but $\Lambda_{f}=\boldsymbol{C}$. This follows by using an analytic curve $(x(t), y(t), z(t)) \in C^{3} \quad$ with $\quad x(t)=t,[z(t)]^{2}=-\frac{5}{9} t^{-6}+\lambda t^{-5}+\mu t^{-4}+\cdots \quad$ and $\quad[y(t)]^{3}=$ $-\frac{5}{2} t^{-6}-\frac{81 \lambda}{4} t^{-5}+\frac{9\left(4-90 \mu-891 \lambda^{2}\right)}{40} t^{-4}+\cdots$.

3. Our conjecture is that for a Newton nondegenerate polynomial $f$ with $f(0)=0$ we have $\bigcup_{\Delta \in \mathscr{B}} \Sigma_{\Delta} \leqq B_{f}$; for the general case of polynomials, we hope that $S_{f} \subseteq B_{f}$. But without a good description of the (all !) fibers $f^{-1}(c)$ it seems that there exists no simple way to prove this.

However, for $n=2$ we have the following:

Proposition 6. Let $f \in C[x, y]$ be a not convenient and Newton nondegenerate polynomial, not depending only of one variable, and such that $f(0)=0$. Then

$$
B_{f}=\Sigma_{f} \cup\{0\} \cup \cup_{\Delta \in \mathscr{B}} \Sigma_{\Delta}
$$

Proof. We prove this proposition in three steps.

Step 1. $\Sigma_{f} \subseteq B_{f}$. The proof is clear.

Step 2. If $f \in \boldsymbol{C}[x, y]$ is a not convenient polynomial with $f(0)=0$, not depending only of one variable, then $0 \in B_{f}$.

Proof. Since $f$ is not convenient, $f$ has the form $f(x, y)=x g(x, y)$ (or $f(x, y)=y g(x, y)$, which case can be analysed similarly).

If $g(0,0)=0$, then $0 \in \Sigma_{f} \subseteq B_{f}$.

If $g(0,0) \neq 0$, then $g(x, y)=a_{0}+a_{1} y+\cdots+a_{m} y^{m}+x \psi(x, y)$ with $a_{0} \neq 0$.

Suppose that there exists $a_{i} \neq 0, i \in\{1, \cdots, m\}$; by an easy computation we obtain that $0 \subseteq \Sigma_{f}$, hence $0 \subseteq B_{f}$. 
Suppose that $a_{i}=0$ for all $i=1, \cdots, m$; hence $f(x, y)=x\left(a_{0}+x \psi(x, y)\right)$ and $\phi(x, y)$ does depend on the variable $y$. The fiber $f^{-1}(0)$ contains a connected component diffeomorphic to $C$. But the generic fiber $f^{-1}(\lambda), \lambda \neq 0$, doesn't contain any contractible component. To see this, suppose that $T$ is a contractible component of $f^{-1}(\lambda), \lambda \neq 0$; consider the projection $\pi$ of $T$ to the $x$-axis. Then the image $\pi(T)$ is equal to $C^{*} \backslash\{$ finite number of points ; hence $T$ is a contractible (branched) covering of $\pi(T)$ with finite fibers and $\pi_{1}(\operatorname{im} \pi) \neq 0$ and is free, contradiction. Consequently $f^{-1}(0)$ and the generic fiber are not diffeomorphic, hence $0 \in B_{f}$.

Step 3. Let $f \in C[x, y]$ be a not convenient and Newton nondegenerate polynomial, not depending only of one variable, and such that $f(0)=0$. If $c \subseteq \bigcup_{\Delta \in \mathscr{B}} \Sigma_{\Delta} \backslash\left(\Sigma_{f} \cup\{0\}\right)$, then $c \in B_{f}$.

Proof. Indeed, in this case the Euler-characteristic of the special fiber $f^{-1}(c)$ and of the generic fiber $f^{-1}\left(c_{\text {gen }}\right)$ differ. The Euler-characteristic of a fiber $f^{-1}(\lambda)$ can be computed by the following formula:

$$
\left.\chi\left(f^{-1}(\lambda)\right)+\# \overline{\left(f^{-1}(\lambda)\right.} \cap H_{\infty}\right)=2-(d-1)(d-2)+\Sigma \mu_{i}(\lambda),
$$

where $\overline{f^{-1}(\lambda)}$ is the projective closure of $f^{-1}(\lambda), H_{\infty}$ is the hyperplane at infinity and $\Sigma \mu_{2}(\lambda)$ is the sum of the Milnor numbers of the singularities of $\overline{f^{-1}(\lambda)}$ at infinity (see [5]).

We show that $\Sigma \mu_{i}(c)>\Sigma \mu_{i}\left(c_{\text {gen }}\right)$. This follows from the followings two lemmas: the first one describes the singularities at infinity and the second one is applied for these singularities.

Lemma 7. Let $f$ be as above and let $d$ be the degree of $f$ and $F(x, y, z)$ the homogeneousated polynomial of $f$. Then only $(1: 0: 0)$ and $(0: 1: 0)$ can be singula rities of $\overline{f^{-1}(\lambda)}$ at infinity. If $(1: 0: 0)$ is a singularity of $\overline{f^{-1}(\lambda)}$ at infinity, then his equation is $g(y, z)=F(1, y, z)-\lambda z^{d}=0$ and the Newton polygon of this singularity can be obtained from the Newton diagram of $f$ in the following way: let $P, R, O$ be the points in the diagram of $f$ with coordinates $(d, 0),(0, d)$ and respectively $(0,0)$. Then the origin in the diagram of $g$ is $P$, the positive semiaxes are $P R$ and $P O$, corresponding to the y-axis and respectively to the z-axis, and $\overline{\operatorname{supp}(g)}$ corresponds to $\tilde{\Gamma}_{-}(f)$. The nondegeneracy of $f$ on the faces of $\tilde{\Gamma}_{-}(f)$ means the nondegeneracy of $g$ on the corresponding faces of $\overline{\operatorname{supp}(g)}$. If $\Delta$ is a bad face of $\overline{\operatorname{supp}(f)}$ giving rise to the face $\bar{\Delta}$ of the Newton polygon of the singularity in 0 of $g$, then the values $\lambda \in \Sigma_{\Delta} \backslash\{0\}$ are exactely the values of $\lambda$ such that the conditions of nondegeneracy on $\bar{\Delta}$ are not fulfilled.

Lemma 8. Let $h \in C[x, y]$ be a convenient polynomial with $\operatorname{grad} h(0)=0$ and let $\Gamma$ be the Newton polygon of $h$ in origin (see [6], [11] for the definition). 
Suppose that $h$ is nondegenerate on all the faces of $\Gamma$, excepting the 1-dimensional face $\Delta$ of $\Gamma$ which has an endpoint on the axis $O x$, where the nondegeneracy condition is not fulfilled. Then the Milnor number $\mu(h, 0)$ of $h$ in 0 is strictly greater than the Newton number $\nu(\Gamma)$. (See [6], [11] for the definition of $\nu(\Gamma)$.)

We omit the proof of Lemma 7 , since it is a straightforward verification. For the proof of Lemma 8 , let $(a, 0)$ and $(b, c)$ be the coordinates of the terminal points of $\Delta$. We consider the covering $\varphi:\left(\mathbb{C}^{2}, 0\right) \rightarrow\left(\mathbb{C}^{2}, 0\right), \varphi(x, y)=\left(x^{c}, y\right)$ and the singularity $h^{\prime}(x, y)=h(\varphi(x, y))$ with Newton polygon $\Gamma^{\prime}$; the relations (15) and (12) from [3] between $\mu\left(h^{\prime}, 0\right)$ and $\mu(h, 0)$, respectively $\nu(\Gamma)$ and $\nu\left(\Gamma^{\prime}\right)$ enable us to suppose that $c$ divides $a-b$. Let $m=\frac{a-b}{c}$. We have $f_{\Delta}=$ $\alpha x^{b}\left(y+\beta_{1} x^{m}\right) \cdots\left(y+\beta_{c} x^{m}\right)$ with $\alpha, \beta_{1}, \cdots, \beta_{c} \in \mathbb{C}^{*}$. The degeneracy condition on $\Delta$ means that there exist $i, j \in\{1, \cdots, c\}, i \neq j$, such that $\beta_{i}=\beta_{j}$. Hence we can consider that $f_{\Delta}=\alpha x^{c}\left(y+\beta_{1} x^{m}\right)^{r} \cdot\left(y+\beta_{r+1} x^{m}\right) \cdot \cdots \cdot\left(y+\beta_{c} x^{m}\right)$ for some $r \geqq 2$. We change the variables: $\tilde{x}=x, \tilde{y}=y+\beta_{1} x^{m}$. Then all the faces of $\Gamma$, excepting $\Delta$, will be faces of the new Newton polygon $\Gamma^{\prime \prime}$ and $\Gamma^{\prime \prime} \supseteqq \Gamma, \Gamma^{\prime \prime} \neq \Gamma$. Now it is easy to see that $\nu\left(\Gamma^{\prime \prime}\right)>\nu(\Gamma)$ and we finish the proof of Lemma 8 using Kouchnirenko's results [6].

Note that the analogue of Lemma 8 for polynomials with $n \geqq 3$ variables in general is not true (see Remarque 1.21 from [6]).

4. Our Proposition 6 (and the inequality from Step 3) can be compared with a result of Hà and Lê (which says that, for $n=2$, a bifurcation point $\lambda$ is either critical point or $\chi\left(f^{-1}(\lambda)\right) \neq \chi\left(f^{-1}\left(c_{\text {gen }}\right)\right.$ ), and a result of M. Suzuki (which says that $\chi\left(f^{-1}(\lambda)\right) \geqq \chi\left(f^{-1}\left(c_{\text {gen }}\right)\right)$, see $[14]$, Théorème 1$)$.

\section{References}

[1] Broughton, S.A., On the topology of polynomial hypersurface, Proceedings of Symposia in Pure Mathematics, 40, Part 1, 1983.

[2] -, Milnor numbers and the topology of polynomial hypersurfaces, Invent. Math., 92 (1988), 217-241.

[3] Dimca, A. and Tibăr, M., Pull-backs of isolated singularities under analytic coverings, Rev. Roum. Math. Pures Appl., 32 (1987), 523-530.

[4] Fedoryuk, M.V., The asymptotics of the Fourier transform of the exponential function of a polynomial, Dokl. Akad. Nauk, 227 (1976), 580-583.

[5] Hà, H.V. and Lê, D. T., Sur la topologie des polynômes complexes, Acta Math. Vietnamica, 9 (1984), 21-32.

[6] Kouchnirenko, A.G., Polyèdres de Newton et nombres de Milnor, Invent. Math., 32 (1976), 1-31.

[7] Milnor, J., Singular Points of Complex Hypersurfaces, Ann. of Math. Studies, 61, Princeton Univ. Press, 1968.

[8] Némethi, A., Théorie de Lefschetz pour les variétés algébriques affines, C.R. Acad. Sc. Paris, 303, Série I, n. 12 (1986).

[9] - Lefschetz theory for complex affine varieties, Rev. Roum. Math. Pures 
Appl., 33 (1988), 233-260.

[10] Némethi, A. and Zaharia, A., Milnor fibration at infinity, in preparation.

[11] Oka, M., On the bifurcation of the multiplicity and topology of the Newton boundary, J. Math. Soc. Japan, 31 (1979), 435-450.

[12] Pham, F., La descente des cols par les onglets de Lefschetz, avec vues sur GaussManin, Systèmes différentiels et singularités, Juin-Juillet 1983, Astérisque 130.

[13] — - Vanishing homologies and the $n$ variable saddlepoint method, Proceedings of Symposia in Pure Mathematics, 40, Part 2, 1983.

[14] Suzuki, M., Propriétés topologiques des polynômes de deux variables complexes, et automorphismes algébriques de l'espace $\boldsymbol{C}^{2}$, J. Math. Soc. Japan, 26 (1974), 241-257.

[15] Varchenko, A.N., Theorems on Topological Equisingularity of Families of Algebraic Varieties and Families of Polynomial Maps, Izvestiya Akad. Nauk, 36 (1972), 9571019.

[16] Verdier, J.L., Stratifications de Whitney et Théorème de Bertini-Sard, Invent. Math., 36 (1976), 295-312. 
